Z. Klin. Chem. Klin. Biochem.

12. Jg. 1974 , S. $504-507$

\title{
Radioimmunologische Aldosteronbestimmung im Urin als einfache Routinemethode ${ }^{1,2}$ )
}

\author{
Von K.A. Deck ${ }^{3}$ ) und L. Eberlein \\ Medizinische Universitätsklinik (Direktor: Prof. Dr. R. Gross) Köln
}

(Eingegangen am 14. Mai/16. August 1974)

Es wird eine einfache radioimmunologische Bestimmungsmethode für die Aldosteronausscheidung im Urin beschrieben, welche bei geringem Aufwand an Arbeit und Gerät zuverlässige Ergebnisse liefert.

\section{A simple routine method for the radioimmunological determination of urinary aldosterone}

A simple routine method for the radioimmunological determination of urinary aldosterone is described, which gives reliable results with minimum demands on manpower and equipment.

Durch die Kopplung von Steroidderivaten an Proteine und die Immunisierung von Versuchstieren mit diesen Konjugaten (1) sind in den vergangenen Jahren Antisera von hoher Spezifität gegen viele Steroide gewonnen worden. Die Verwendung dieser Antisera in radioimmunologischen Assays erlaubt bei genügender Spezifität die Messung von Steroidmengen im Picogrammbereich ohne vorherige chromatographische Reinigung des biologischen Materials. Eine Überprüfung der Spezifität solcher Methoden durch Einschalten von Chromatographien oder Kontrolle mit Methoden, deren Richtigkeit erwiesen ist, sollte jedoch stets vorgenommen werden, was oft nicht berücksichtigt wird.

Da Aldosteron im Vergleich zu anderen Steroiden in biologischen Flüssigkeiten in geringer Konzentration vorkommt, wird in vielen der bisher angegebenen radioimmunologischen Aldosteronbestimmungsmethoden der immunologischen Reaktion eine Chromatographie oder Gelfiltration vorgeschaltet, um bei der AntikörperSteroidbindungsreaktion eine Kreuzreaktion mit höher konzentrierten, anderen Steroiden zu vermeiden (2-7). Whalen et al. (8) gaben erstmals eine radioimmunologische Aldosteronbestimmungsmethode an, bei der auf chromatographische Reinigung verzichtet wird.

Dieses Verfahren beruht auf folgendem Prinzip: Der von den meisten Autoren im Urin gemessene Metabolit des Aldosterons, das 18-Aldosteronglucuronid, wird

1) Mit Unterstützung der Deutschen Forschungsgemeinschaft.

2) Eine vorläufige Mitteilung erschien in l.c. (9).

3) Vorarbeiten zur Entwicklung der Methode wurden in der Div. of Endocrinology, University of Michigan Medical Center, Ann Arbor, U.S.A., durchgeführt. zunächst durch Säurehydrolyse bei Raumtemperatur aus der Glucuronidbindung freigesetżt, dann extrahiert und weiter aufgearbeitet. Durch die Säurehydrolyșe bei Raumtemperatur werden offensichtlich keine anderen Steroide aus Konjugatbindungen in genügend großen Mengen freigesetzt, um mit spezifischen Aldosteronantikörpern in nennenswertem Ausmaß zu reagieren. Entfernt man also vor der Säurehydrolyse durch Präextraktion alle freien Steroide aus dem Urin, so läßt sich nach der Hydrolyse das Aldosteron in einer für die immunologische Reaktion genügend gereinigten Form extrahieren. Whalen et al.(8) haben ihre Ergebnisse nicht durch Einschaltung einer Chromatographie oder Anwendung der Doppelisotopenmethode geprüft; außerdem wird in ihrer Methode mehrfach extrahiert, transferiert und aliquotiert, weshalb radioaktiv markiertes Aldosteron dem Urin zu Beginn als Verlustmarkierung zugesetzt werden muß. Durch żweckmäßige Verringerung der Anzahl der Aufarbeitungsschritte und Verwendung eines speziellen Extraktionsgefäßes (Abb. 1) ließ sich das Verfahren so modifizieren (9), daß eine Wiederfindung von etwa 90-95\% erreicht wird, der Aufwand an Arbeit und Glasgeräten wesentlich reduziert, die Verlustmarkierung entbehrlich und die Methode besser für Routinezwecke adaptiert wird. Sie soll deshalb im folgenden dargestellt werden.

\section{Methodik}

\section{Reagenzien}

Methanol, Methylenchlorid und Äthanol (Merck), über Kieselgel gereinigt, redestilliert.

Pufferlösung: $8,17 \mathrm{~g} \mathrm{NaCl}, 6,86 \mathrm{ml} \mathrm{0,5} \mathrm{mol} / 1 \mathrm{NaH}_{2} \mathrm{PO}_{4}, 13,71 \mathrm{ml}$ $0,5 \mathrm{~mol} / 1 \mathrm{Na}_{2} \mathrm{HPO}_{4}, 0,1 \mathrm{~g}$ Merthiolate (Serva), bzw. $10 \mathrm{ml}$ 


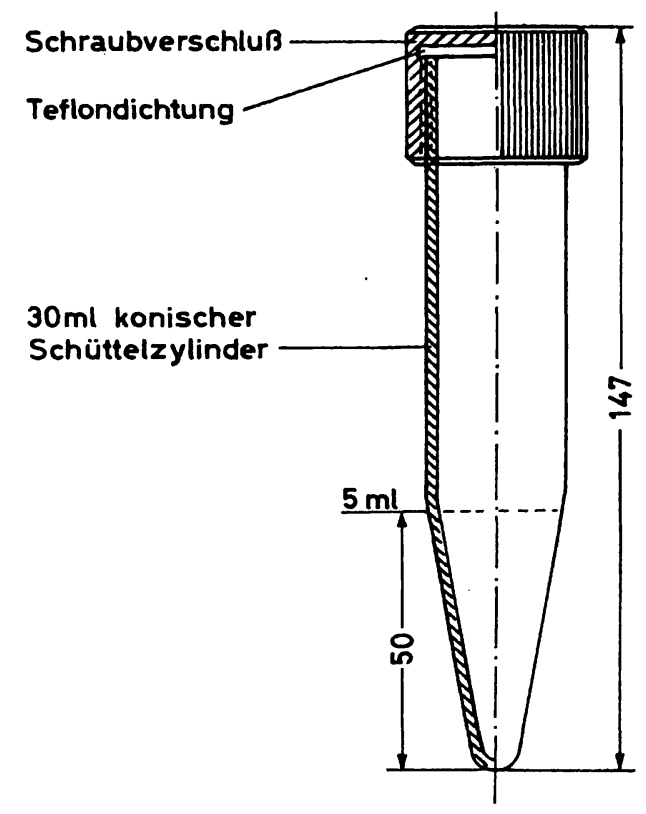

Abb. 1. Schüttelzylinder zur Aufarbeitung der Urinproben. Maße in $\mathrm{mm}$.

einer $10 \mathrm{~g} / \mathrm{l}$ Merthiolate-Lösung, mit bidest. Wasser auf 11 auffüllen und auf $\mathrm{pH} 7$ einstellen.

Gelatine-Lösung, $1 \mathrm{~g} / \mathrm{l}:$ 0,25 $\mathrm{g}$ Gelatine (Serva), $2,5 \mathrm{ml} 10 \mathrm{~g} / \mathrm{l}$ Merthiolate-Lösung, $250 \mathrm{ml}$ Pufferlösung.

Gelatine-Lösung, $5 \mathrm{~g} / \mathrm{l}: 1,25 \mathrm{~g}$ Gelatine, $2.5 \mathrm{ml} 10 \mathrm{~g} / 1$ Merthiolate-Lösung, $250 \mathrm{ml}$ Pufferlösung.

Szintillator: $21 \mathrm{~g}$ PPO (Packard), 0,9 g POPOP, 21 Toluol, 2-3 h rühren, dann zugeben $500 \mathrm{ml}$ Triton X 100 (Decryl Chemie Frankfurt), $500 \mathrm{ml}$ Triton X 45 .

Kohlelösung: $100 \mathrm{ml} \mathrm{H} 2 \mathrm{O}+250 \mathrm{mg}$ Dextran T 70 (Pharmacia) lösen, $+250 \mathrm{mg}$ Kohle (Norit A, Serva), 1-2 h rühren.

Antiserum: 1:200 000 verdünnt mit $10 \mathrm{~g} / 1$ Rinderserumalbumin (Behring, trocken, reinst) in $1 \mathrm{~g} / 1$ Gelatine-Pufferlösung. Die Charakterisierung dieses Antiserums und Gewinnung von Standardkurven wurde an anderer Stelle beschrieben (4).

Standards: $10 \mathrm{mg}$ Aldosteron (Merck) werden in $50 \mathrm{ml}$ Äthanol aufgenommen, und die Konzentration fotometrisch bestimmt. Durch entsprechende Verdünnungsschritte werden Standards mit Konzentrationen von $2000,1000,500,250,125$ und $62,5 \mu \mathrm{g} / \mathrm{l}$ (entsprechend $200-6,25 \mathrm{pg} / 0,1 \mathrm{ml}$ ) hergestellt.

$3 \beta-5 \alpha$-Tetrahydroaldosteron (Ikapharm, Israel)

Lösung mit tritiummarkierten Aldosteron: $\left[1,2-\mathrm{H}^{3}\right]-D$-Aldosteron, $54 \mathrm{Ci} / \mathrm{mmol}$ (NEN), $250 \mu \mathrm{g}$ gelöst in $50 \mathrm{ml}$ Äthanol, 1:500 mit bidest. Wasser verdünnt.

\section{Gerät}

Alle Glasgeräte müssen säuregewaschen sein, sie werden jeweils nach Gebrauch mit tridest. Wasser, Methanol und Aceton gespïlt und immer fï den gleichen $Z$ weck verwendet. Verwendung von Eppendorfpipetten, Einmal-Pasteurr-Pipetten und Einmalreagenzgläsern.

\section{Aufarbeitung der Urinproben}

$0,5 \mathrm{ml}$ Urin und $5 \mathrm{ml}$ tridest. Wasser werden in einem konischen, durch Schraubverschluß verschließbaren, speziell angefertigten Schüttelzylinder (Abb. 1) mit $20 \mathrm{ml}$ Methylenchlorid durch fünfminütiges Schütteln auf der Schüttelmaschine präextrahiert. Anschließend Zentrifugation für $10 \mathrm{~min}$ bei $1000 \mathrm{U} / \mathrm{min}$, Absaugen der Methylenchlorid-Unterphase mit einer Einmal-PasteurPipette; dabei muß der Gummischlauch, welcher die Pipette mit der Wasserstrahlpumpe verbindet, während des Durchstechens der wäßrigen Phase mit dem Finger abgeklemmt werden. Die verbleibende wäßrige Phase sinkt in den konischen Teil des Gefäßes, wird zur Säurehydrolyse am pH-Meter auf pH 1 eingestellt und $24 \mathrm{~h}$ bei Raumtemperatur inkubiert. Danach erfolgt eine zweite Extraktion mit $20 \mathrm{ml}$ Methylenchlorid, Absaugen der wäßrigen Phase und Waschen des Extraktes mit $5 \mathrm{ml} \mathrm{0,1} \mathrm{mol} / 1 \mathrm{NaOH}$-Lösung, anschließend mit $0,1 \mathrm{~mol} / 1 \mathrm{Essig}$ säure und zweimal mit $5 \mathrm{ml}$ tridest. Wasser. Nahezu quantitatives Absaugen der Oberphase erst nach dem letzten Waschvorgang. Abdampfen des Methylenchlorids zur Trockne, Aufnahme des Rückstands in $10 \mathrm{ml}$ Methanol, danach Überführung von je zwei mal 0,1 ml Methanol in Einmalreagenzgläser $(72 \times 12 \mathrm{~mm})$, Abdampfen des Methanols im Vakuum bei $40^{\circ}$.

Radioimmunologische Bestimmung

Zugabe von $0,1 \mathrm{ml}$ der $1 \mathrm{~g} / 1$ Gelatine-Phosphatpufferlösung zu den Trockenrückständen von Proben, Standards und Leerwerten, sowie von $1 \mathrm{nCi}$ tritiummarkierten Aldosterons in $0,1 \mathrm{ml}$ wäßriger Lösung. Anschließend Zugabe von $0,2 \mathrm{ml}$ der Antiserumlösung. Das Gemisch wird etwa $30 \mathrm{~s}$ auf dem Vortex-Mixer geschüttelt und bei $4^{\circ} \mathrm{C}$ für mindestens $6 \mathrm{~h}$ inkubiert. Die Trennung des freien vom antikörpergebundenen Aldosterons erfolgt durch Zugabe einer Suspension von $0,5 \mathrm{ml}$ dextranbeschichtetem Norit-A, Schütteln, Inkubation bei $4^{\circ}$ für $10 \mathrm{~min}$, Zentrifugation in der Kühlzentrifuge und Dekantieren des Überstands von der sedimentierten Kohle ins Zählglas. Zählung im PackardSzintillationszähler.

\section{Ergebnisse}

\section{Methodische Kriterien \\ Wiederfindung}

Der Verlust von Aldosteron während der Reinigungsphase wurde durch Zusatz von markiertem Aldosteron nach Präextraktion und Messung eines Aliquots aus dem Methanol nach Aufnahme des Rückstandes bestimmt. Bei $n=240$ Proben wurde eine Wiederfindung von 94,6 $\pm 5,8 \%$ erzielt; ein interner Standard ist also bei der verwendeten Methode entbehrlich.

\section{Genauigkeit}

Die Intra-Assay-Streuung war $8,4 \pm 0,46$ und $8,2 \pm 0,35$ $\mu \mathrm{g} / 24 \mathrm{~h}$ bei Bestimmung von $\mathrm{n}=8$, bzw. $\mathrm{n}=7$ Proben aus zwei verschiedenen Urinen. Die Inter-Assay-Variation wurde durch komplette Aufarbeitung von Proben aus dem gleichen Urin in 21 Serien bestimmt; der Variationskoeffizient war $14,1 \%$. Wurde eine Pufferlösung statt des Urins aufgearbeitet, so resultierte unter der Annahme einer täglichen Urinausscheidung von 1,5 1 ein Leerwert von $0,5 \mu \mathrm{g} / 24 \mathrm{~h}$.

\section{Richtigkeit}

Die Spezifität der Methode wurde überprüft durch Feststellung der Kreuzreaktion des Antiserums mit anderen Steroiden, Einschaltung einer Chromatographie im Bush B 5 System nach der zweiten Extraktion, und Vergleich der Ergebnisse der radioimmunologischen $\mathrm{Be}-$ stimmung mit Ergebnissen aus den gleichen Urinproben nach der Doppelisotopenmethode von Kliman \& Peterson (11), deren Richtigkeit im Verlauf der vergangenen 15 Jahre von vielen Autoren nachgewiesen wurde.

Die Kreuzreaktion des Antiserums mit einigen Steroiden wurde nach Abraham (13) geprüft und berechnet und betrug: Östriol $<0,001 \%$, Östradiol 0,010\%, Dehydro- 
epiandrosteron 0,012\%, Androstendiol 0,013\%, Cortisol $0,030 \%$, Testosteron $0,060 \%$, Androstendion $0,100 \%$, Corticosteron $0,150 \%$, Cortison $0,200 \%$, Progesteron 0,250\%, Desoxycorticosteron 0,375\%, Tetrahydroaldosteron $10 \%$.

Die Ergebnisse der chromatographierten Proben waren im Mittel 93,6 \pm 17,3\% der entsprechenden nicht chromatographierten Proben (s. Tabelle 1).

Bei 22 Personen waren die Ergebnisse der radioimmunologischen und der Doppelisotopenmethode statistisch signifikant linear korreliert (Regressionsgrade $y=0,901$, $\mathrm{x}=0,368, \mathrm{r}=0,94, \mathrm{P}<0,001)$.

Tab. 1. Aldosteronbestimmung im Urin

a) Ergebnis der Bestimmung in $\mu g / 1$ Urin, ohne internen Standard und Chromatographie.

b) Ergebnis der Bestimmung in $\mu g / 1$ Urin mit eingeschalteter Chromatographie und Benutzung eines internen Standards.

\begin{tabular}{|c|c|c|c|}
\hline Probe Nr. & (a) & (b) & $\begin{array}{c}b \times 100 \\
a \\
\end{array}$ \\
\hline $\begin{array}{r}1 \\
2 \\
3 \\
4 \\
5 \\
6 \\
7 \\
8 \\
9 \\
10 \\
11 \\
12 \\
13 \\
14 \\
15 \\
16 \\
17 \\
18 \\
19 \\
20 \\
21 \\
22 \\
23 \\
24 \\
25 \\
26 \\
27 \\
28 \\
29 \\
30 \\
31 \\
32\end{array}$ & $\begin{array}{r}4,1 \\
4,9 \\
5,2 \\
5,5 \\
6,0 \\
6,2 \\
6,2 \\
6,6 \\
6,8 \\
6,9 \\
7,0 \\
7,2 \\
7,2 \\
7,1 \\
7,5 \\
7,6 \\
7,8 \\
7,9 \\
7,9 \\
8,0 \\
8,1 \\
8,3 \\
8,4 \\
8,6 \\
8,6 \\
8,6 \\
9,1 \\
9,4 \\
9,7 \\
9,8 \\
10,1 \\
10,4\end{array}$ & $\begin{array}{r}3,5 \\
3,7 \\
5,1 \\
5,9 \\
3,5 \\
5,5 \\
4,7 \\
6,9 \\
8,0 \\
6,9 \\
5,7 \\
7,5 \\
4,9 \\
7,5 \\
8,1 \\
7,3 \\
5,1 \\
7,7 \\
7,5 \\
9,4 \\
6,0 \\
7,6 \\
9,4 \\
6,9 \\
5,4 \\
10,6 \\
9,4 \\
8,9 \\
11,0 \\
8,2 \\
11,5 \\
9,7\end{array}$ & $\begin{array}{r}85,4 \\
75,5 \\
98,0 \\
107,3 \\
58,3 \\
88,7 \\
75,8 \\
104,5 \\
117,6 \\
100,0 \\
81,4 \\
104,2 \\
68,0 \\
102,7 \\
108,0 \\
96,1 \\
65,4 \\
97,5 \\
94,9 \\
117,5 \\
74,0 \\
91,5 \\
111,9 \\
80,2 \\
62,8 \\
123,3 \\
103,4 \\
94,6 \\
113,4 \\
83,6 \\
113,9 \\
93,2 \\
\end{array}$ \\
\hline & & & $\begin{array}{l}=93,5 \\
\pm 17,3\end{array}$ \\
\hline
\end{tabular}

\section{Normalwerte}

Bei 10 Normalpersonen mit unbeschränkter Kost fand sich radioimmunologisch ein Mittelwert von $7,1 \pm 1,3 \mu \mathrm{g}$ /Tag, mit der Doppelisotopenmethode ein Mittelwert von $7,8 \pm 2,4 \mu \mathrm{g} / \mathrm{T}$ ag.

Bei 12 Normalpersonen mit einer Diät mit $120 \mathrm{mmol}$ $\mathrm{NaCl} / \mathrm{Tag}$ war die mittlere Ausscheidung radioimmunologisch gemessen 10,3 $\pm 2,5 \mu \mathrm{g} / \mathrm{Tag}$, mit der Doppelisotopenmethode $11,1 \pm 3,1 \mu \mathrm{g} / \mathrm{Tag}$. Bei einem weiteren Kollektiv von 15 Normalpersonen bei freier Kost wurde eine mittlere Ausscheidung von 8,2 $\pm 2,1 \mu \mathrm{g} /$ Tag mit der radioimmunologischen Methode ermittelt.

Bei 4 Patienten mit primärem Hyperaldosteronismus lagen unter normalen diätetischen Bedingungen Aldosteron-Ausscheidungswerte zwischen 30 und $125 \mu \mathrm{g} /$ Tag vor; bei insgesamt 10 Patienten mit sekundärem Hyperaldosteronismus (dekompensierte Lebercirrhose) war die Ausscheidung zwischen 20 und $155 \mu \mathrm{g} / \mathrm{Tag}$.

\section{Diskussion}

Die beschriebene Methode ist einfach und spezifisch; sie ergibt in unseren und auch anderen Laboratorien (12) Werte, die mit den Doppelisotopenmethode-Werten gut korreliert sind und sich durch Einschaltung einer Chromatographie nicht wesentlich verändern. Die Methode ist sehr wenig arbeitsaufwendig, da eine technische Assistentin mit einem Gesamtarbeitsaufwand von etwa 8 Stunden 36 Proben aufârbeiten kann.

Beim Vergleich radioimmunologischer Bestimmungsmethoden muß berücksichtigt werden, daß verschiedene Antiseren verschiedene Spezifitäts- und Sensibilitätscharakteristika haben. Das von uns verwendete Antiserum $(9,10)$ ist offensichtlich spezifisch genuig, um mit der durch Säurehydrolyse bei Raumtemperatur freigesetz̈ten, bisher nicht näher identifizierten corticosteroidähnlichen Aktivität (14) nicht kreuzzureagieren. Diese corticoidähnliche Aktivität wurde mit der sehr unspezifischen Methode von Murphy \& Pattee (15) nachgewiesen; es liegt die Vermutung nahe, daß es sich dabei um dekonjugierte Tetrahydroderivate der Corticosteroide handelt, die infolge der Tetrahydrokonstellation nur geringfügig mit dem Antikörper reagieren. Tatsächlich weisen jạ auch einige entsprechend untersuchte Aldosteronantiseren (14) eine relativ geringe Kreuzreaktion mit Tetrahydroaldosteron auf, die bei Tetrahydroderivaten anderer Corticoide wahrscheinlich noch geringer ist.

\section{Literaturverzeichnis}

1. Erlanger, B. F., Borek, F., Beiser, S. M. \& Liebermann, S. (1957), J. Biol. Chem. 288, 713-727.

2. Bayard, F., Beitins, I. Z., Kowarski, A. \& Migeon, C. J. (1970), J. Clin. Endocrinol. Metab. 31, 1-6.
3. Ito, T., Woo, J., Haning, R. \& Horton, R. (1972), J. Clin. Endocrinol Metab. 34, 106-112.

4. Mayes, D., Furuyama, S., Kem, D. C. \& Nugent, C. A. (1970), J. Clin. Endocrinol. Metab. 30, 682-685. 
5. McCaa, R. E., McCaa, C. S., Read, D. G., Bower, J. D. \& Guyton, A. C. (1972), Circulat. Res. 31, 473-480.

6. Vescei, P., Benke, B., Joumach, A., (1972), Experientia 28, $622-624$.

7. Waldhäusl, W., Haydl, H. \& Frischauf, H. (1972), Steroids 20,727-736.

8. Whalen, J. D., Tyler, F. H., West, C. D., (1970), Clin. Res. $18,172$.

9. Deck, K. A., Verhandlg. (1972), Deut. Ges. Inn. Med. 78, 1605-1607.

10. Deck, K. A., Champion, P. K., Conn., J. W., (1973), J. Clin. Endocrinol. Metab. 36, 756-760.
11. Kliman, B. \& Peterson, R. E. (1960), J. Biol. Chem. 235, 1639-1648.

12. Conn, J. W., Cohen, E. L., Lucas, C. D., McDonald, W. J., Mayer, G. H., Blough, W. M., Eveland, W. C., Bookstein, J. J., Lapides, J. (1972), Arch. Int. Med. 130, 682-696.

13. Abraham, G. E. (1969), J. Clin. Endocrinol. Metab. 29, 866-870.

14. Vetter, W., Vetter, H., Siegenthaler, W. (1973), Acta Endocrinol. 74, 558-567.

15. Murphy, B. E. P. \& Pattee, C. J. (1964), J. Clin. Endocrinol. Metab. 24, 919-923.
PD Dr. K. Deck

Oberarzt

Med. Univ.-Klinik

5 Köln 41

Joseph-Stelzmann-Str. 6 